\title{
Tailoring the Optical Dipole Force for Molecules by Field-Induced Alignment
}

\author{
S. M. Purcell and P.F. Barker \\ Department of Physics and Astronomy, University College London, London, WCIE 6BT, United Kingdom
}

(Received 17 March 2009; revised manuscript received 7 June 2009; published 5 October 2009)

\begin{abstract}
We report on the ability to tailor the optical dipole force for molecules by tuning their effective polarizability with strong field alignment using polarized fields. We have measured a difference of $20 \%$ in the dipole force on cold $\mathrm{CS}_{2}$ molecules when changing from linear to near-circular polarization using peak field intensities of $5.7 \times 10^{11} \mathrm{~W} \mathrm{~cm}^{-2}$. A variation in the focal length with laser polarization of a molecular-optical lens formed by a single focused laser beam was also measured. This provides a new way of modifying this force for many molecules.
\end{abstract}

DOI: 10.1103/PhysRevLett.103.153001

The optical dipole force has become an important tool for the manipulation of particles at micron length scales and below. It has extensive applications in ultracold atomic and molecular physics [1] and atom optics [2,3]. It is also central to the operation of optical tweezers [4] and its applications in biology [5] and chemistry [6]. It results from an induced dipole potential created by the interaction between the optical field and the dipole moment induced in the particle by the same field. The force is proportional to the polarizability of the particle and the gradient in the potential. Many molecules and larger particles have a nonspherical shape for which the induced dipole moment and the dipole force are dependent on the degree of orientation or alignment of a molecular axis with respect to the field polarization. Linear molecules have a larger polarizability along the molecular axis when compared to the polarizability perpendicular to it, and the dipole force will be strongest when the molecular axis is aligned with the field polarization. This variation in the dipole force with molecular orientation is not observed in the gas phase due to the random orientations of molecules. In strong fields $\left(10^{12} \mathrm{~W} \mathrm{~cm}^{-2}\right)$, however, rotational motion can be driven in a well-controlled way, leading to an alignment of the molecular axis with the field polarization resulting in molecular alignment [7-10]. This significantly reduces the deleterious effects of orientational averaging in measurements of an ensemble of molecules [11]. Molecular alignment has been used for imaging of molecular orbitals [12], and for measuring time-resolved molecular frame dynamics [13]. Molecular alignment is also seen as an important ingredient for diffractive imaging of single molecules $[14,15]$. The use of strong optical fields therefore allows the simultaneous control of molecular alignment as well as the center-of-mass motion via the dipole force. The dipole force has been used to manipulate the center-of-mass motion of molecules in strong optical fields demonstrating molecular Stark acceleration and deceleration [16], a molecular prism [17], and a lens [1,18-20]. Such strong fields produce large optical Stark potentials in the $100 \mathrm{~K}$ range [21], allowing manipulation on nanosecond time scales. However, the observation of the effect of laser-induced
PACS numbers: $37.10 . \mathrm{Vz}, 33.15 .-\mathrm{e}, 42.50 . \mathrm{Hz}, 42.50 . \mathrm{Tx}$

alignment on the dipole force, although predicted [22], has not been reported. In this Letter, we describe the modification of the dipole force on carbon disulfide $\left(\mathrm{CS}_{2}\right)$ molecules, due to field-induced alignment within a strong laser field. We show that the dipole force can be tuned by changing from linear and to a near-circularly polarized field.

The induced dipole (Stark) potential of a molecule irradiated by an optical pulse of electric field $\mathbf{E}(r, t)=$ $\frac{1}{2} \hat{\varepsilon} E(r, t) e^{i \omega_{l} t}+$ c.c. is given by $V(r, t)=-\frac{1}{4} \alpha_{\text {eff }} E(r, t)^{2}$ [1], where $\hat{\varepsilon}$ is a unit vector of the field polarization, $E(r, t)$ is its amplitude, and $\omega_{l}$ its frequency. For linearly polarized light incident on a linear or symmetric top molecule, the effective polarizability is $\alpha_{\text {eff }}=\left[\Delta \alpha \cos ^{2} \theta_{\text {lin }}+\alpha_{\perp}\right]$, where $\Delta \alpha=\alpha_{\|}-\alpha_{\perp}$ and $\alpha_{\|}$and $\alpha_{\perp}$ are the parallel and perpendicular polarizabilities with respect to the molecular symmetry axis. For the linear molecule, $\mathrm{CS}_{2}$, the symmetry axis is along the bond axis, and $\theta_{\text {lin }}$ is the angle between this axis and a laboratory fixed direction defined to be along the polarization vector for linearly polarized light. For circularly polarized light, the effective polarizability is $\alpha_{\text {eff }}=\frac{1}{2}\left[\alpha_{\perp}+\alpha_{\|}-\Delta \alpha \cos ^{2} \theta_{\text {circ }}\right]$, where $\theta_{\text {circ }}$ is the angle between the molecular axis and the laboratory fixed axis along the laser propagation direction. For linearly polarized fields the maximum force is determined by the maximum effective polarizability when $\cos ^{2} \theta_{\operatorname{lin}}=1$ and the molecule is aligned with the polarization vector with $\alpha_{\text {eff }}=\alpha_{\|}$. For circularly polarized light, the maximum polarizability occurs when $\cos ^{2} \theta_{\text {circ }}=0$ and the molecule moves in the plane of the polarization vector (i.e., perpendicular to the propagation direction) with maximum polarizability $\alpha_{\text {eff }}=\left[\alpha_{\perp}+\alpha_{\|}\right] / 2$. We determine the expectation value, $\left\langle\cos ^{2} \theta_{\text {lin } / \text { circ }}\right\rangle$, and the effective polarizability by solving the Schrödinger equation for a rigid rotor. The Hamiltonian is $\mathbf{H}=\mathbf{J}^{2}+V(r, t)$, where $\mathbf{J}$ is the angular momentum operator. The aligned molecule wave function, $\Psi(t)=\sum_{J M} C_{J, M}(t)|J M\rangle$, is a superposition of the field free rotor wave functions $|J M\rangle$, where $J$ is the quantum number for angular momentum and $M$ is its projection onto a space fixed axis. Substitution into the 
Schrödinger equation yields the equations of motion for $C_{J, M}(t)$ [23-25]. The expectation value $\left\langle\cos ^{2} \theta_{\text {lin/circ }}\right\rangle$ is averaged over the thermal Boltzmann distribution to produce an average effective polarizability, $\bar{\alpha}_{\text {eff }}$. When the field evolves slowly compared to the rotational period, alignment adiabatically follows the field [8]. Under this assumption we determine the average effective polarizability for $\mathrm{CS}_{2}$ molecules for the laser intensity and rotational temperature, $T_{R}$, at any spatial location. The static polarizabilities of $\mathrm{CS}_{2}$ are $\alpha_{\|}=16.8 \times 10^{-40} \mathrm{C} \mathrm{m}^{2} \mathrm{~V}^{-1}$ and $\alpha_{\perp}=6.2 \times 10^{-40} \mathrm{C} \mathrm{m}^{2} \mathrm{~V}^{-1}$ [19] and the rotational constant $B=0.109 \mathrm{~cm}^{-1}$ [26]. Figure 1 is a plot of the average effective polarizability for linearly and circularly polarized light at an intensity of $5.7 \times 10^{11} \mathrm{~W} \mathrm{~cm}^{-2}$ as a function of rotational temperature. The average effective polarizability decreases with increasing rotational temperature, and at very high temperatures these values tend towards the field free average polarizability and the dipole force becomes independent of field polarization. The average effective polarizability (inset graph) is calculated for $T_{R}=2,12$, and $35 \mathrm{~K}$, with intensities up to $10^{12} \mathrm{~W} \mathrm{~cm}^{-2}$. At the highest intensities, the effective polarizability saturates.

We have measured the velocity imparted to $\mathrm{CS}_{2}$ molecules for two different field polarizations. A cold beam of $\mathrm{CS}_{2}$ molecules is produced by expanding $7 \mathrm{mbar}$ of $\mathrm{CS}_{2}$ in 1800 mbar of argon into a vacuum chamber. This beam intersects with a pulsed optical field of intensity within a time-of-flight (TOF) mass spectrometer as shown in Fig. 2.

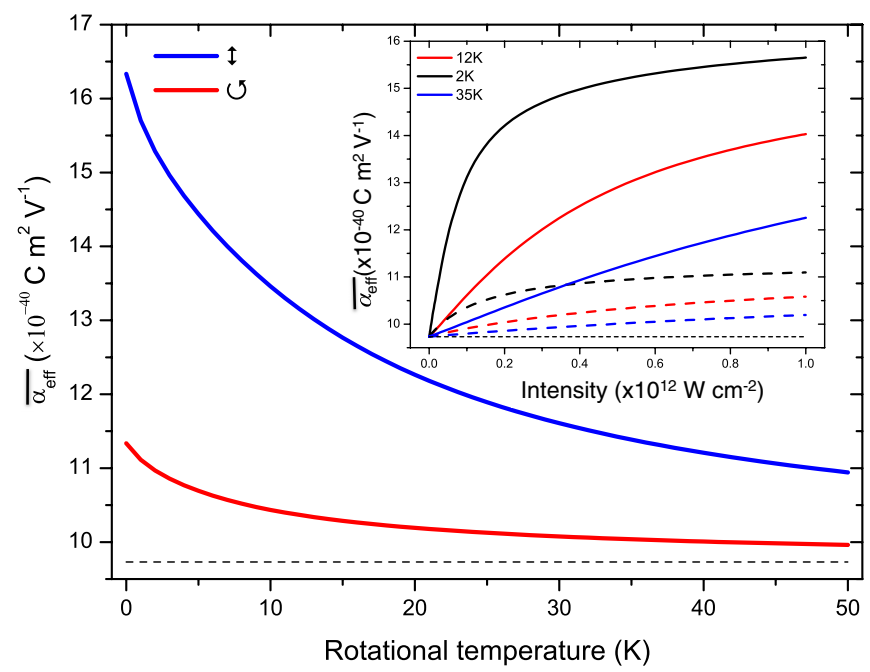

FIG. 1 (color online). Effective polarizability of $\mathrm{CS}_{2}$ for linearly polarized [dark gray (blue) line] and circularly polarized light [light gray (red) line] as a function of temperature at an intensity of $5.7 \times 10^{11} \mathrm{~W} \mathrm{~cm}^{-2}$. The inset graph shows the average effective polarizability of $\mathrm{CS}_{2}$ as a function of intensity calculated for $T_{R}=2 \mathrm{~K}$ (black), $12 \mathrm{~K}$ [light gray (red)], and $35 \mathrm{~K}$ [dark gray (blue)]. The solid lines are for linearly polarized light, and the dashed curves are for circularly polarized light. The average polarizability for randomly oriented molecules is shown by the horizontal short dashed line.
The IR field $(\lambda=1064 \mathrm{~nm})$ is produced by an injectionseeded, $Q$-switched Nd:YAG laser of $\sim 15$ ns duration. The beam is focused to a near Gaussian spatial profile with a $e^{-2}$ radius of $20 \mu \mathrm{m}$ and linear polarization of better than $1: 10^{4}$ along the $y$ direction. The field is converted to circular polarization by a $\frac{\lambda}{4}$ wave plate. The laser energy drops by $6 \%$ after passage through all optics when changing from linear to circular polarization and becomes slightly elliptically polarized. We calculate peak intensities of $7.6 \pm 2.3 \times 10^{11} \mathrm{~W} \mathrm{~cm}^{-2}$ for the linear polarized light and $7.1 \pm 2.2 \times 10^{11} \mathrm{~W} \mathrm{~cm}^{-2}$ for near-circular polarization. To measure the velocity induced by the dipole force we ionize the molecules and measure their TOF once the IR field is turned off. TOF measurements are made by moving the IR field along the $x$ axis while keeping the probe beam fixed. This ensures that any change in the TOF is due only to the variation in dipole force. Each measurement corresponds to a 1200 shot average. The velocity is determined by converting the change in TOF to a change in velocity. Molecules are ionized by a $(3+1)$ resonance enhanced multiphoton ionization scheme via the $\left[\frac{1}{2}\right] n p \sigma_{u}\left({ }^{1} \Pi_{u}\right) \leftarrow X^{1} \Sigma_{g}^{+} \quad$ transition using light at $477.95 \mathrm{~nm}[27,28]$, and $7 \mathrm{~ns}$ in duration, focused to a $e^{-2}$ spot size of less than $5 \mu \mathrm{m}$ with an intensity of $2 \times$ $10^{10} \mathrm{~W} \mathrm{~cm}^{-2}$. Figure $3(\mathrm{a})$ shows the TOF distribution functions for both linear and near-circular polarization when the probe beam was $x=11 \mu \mathrm{m}$ from the center of the IR beam. Changes in TOF are referenced to the center of the distribution when no IR field was present. The TOF signals were recorded $9.3 \mu \mathrm{s}$ after ionization of the molecules. Both polarizations produce a delayed TOF corresponding to deceleration of molecules with a $1.7 \mathrm{~ns}$ time difference between the distributions for each polarization.

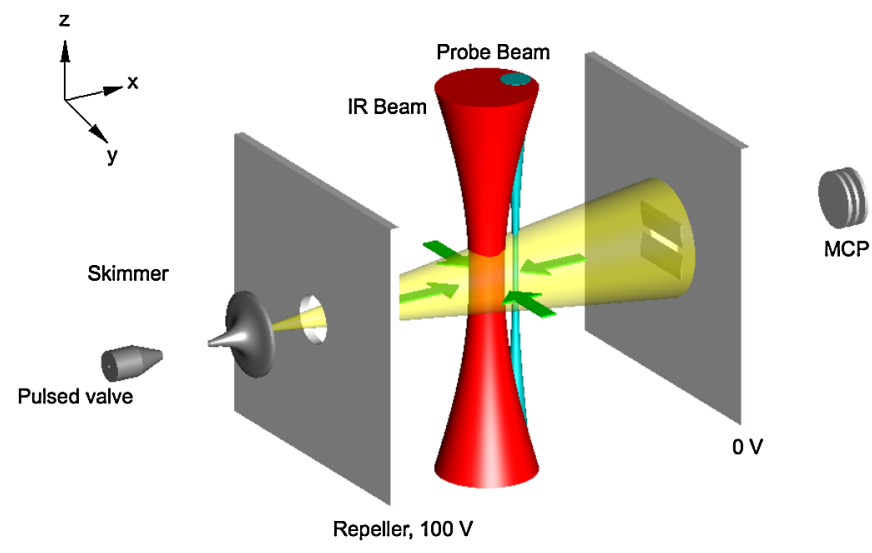

FIG. 2 (color online). The experimental setup used to measure the velocity of $\mathrm{CS}_{2}$ within a time-of-flight mass spectrometer along the $x$ axis. The molecular beam propagates along the $x$ axis where it is crossed by a focused nonresonant laser beam (colored red) and is subject to the dipole force. The molecules experience a force along the $x$ and $y$ axis, shown as the green arrows. Velocity changes along the $x$ axis are detected by ionizing the molecules using a probe beam (colored blue) and recording their time-of-flight on a microchannel plate (MCP). 

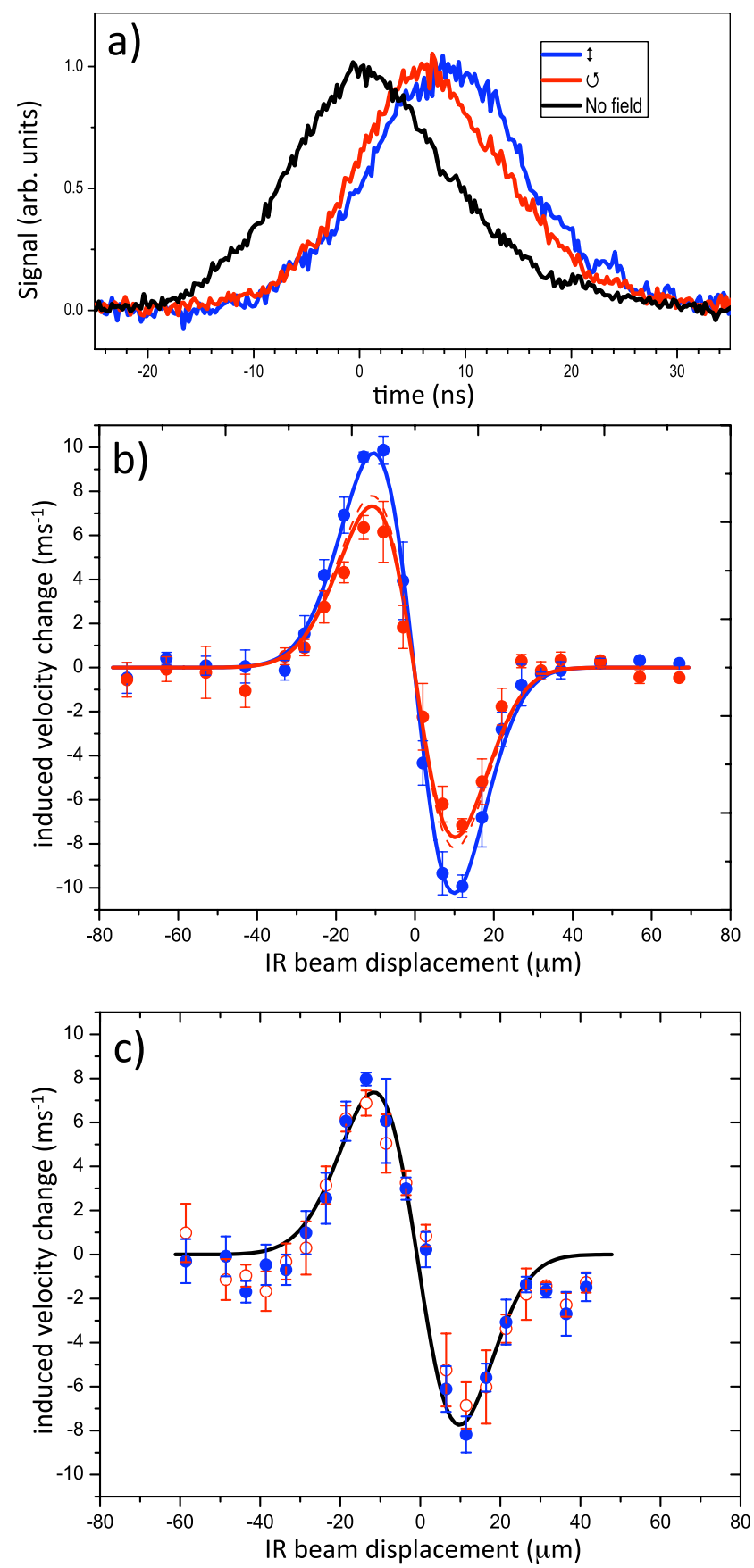

FIG. 3 (color online). (a) Typical TOF distribution of molecules ionized by the UV probe beam $11 \mu \mathrm{m}$ downstream from the center of the IR laser. The black curve is the TOF distribution with no IR field. The dark gray/blue (linear polarized light) and light gray/red (circular polarized light) distributions show the difference between the two polarizations. (b) Measurements of velocity along the $x$ axis of the IR beam. Dark gray (blue) circles are for linearly polarized light and light gray (red) circles for near-circularly polarized light. Solid curves are best fits for linearly and circularly polarized light at $35 \mathrm{~K}$. The dashed curve simulates the effect of increasing the intensity of the circularly polarized beam by $6 \%$ to be equal to the linearly polarized beam. (c) Measurements of molecular velocity in a hot beam. Dark gray (blue) circles are measurements for linearly polarized light and light gray (red) circles for near-circularly polarized light.
As the probe is scanned along the $x$ axis, each TOF distribution function is used to calculate the induced velocity change shown in Fig. 3(b). The velocity shift versus position for both polarizations shows that the molecules are forced towards the center of the IR beam and the peak shifts occur near the maximum gradient of the Gaussian beam [1]. A peak velocity shift of $10 \mathrm{~ms}^{-1}$ is measured for linearly polarized light and $7.3 \mathrm{~ms}^{-1}$ for circularly polarized light at approximately $x= \pm 10 \mu \mathrm{m}$, and the uncertainties are significantly smaller than the shift between the two polarizations. The peak induced velocity and thus the dipole force differ by approximately $25 \%$ when changing from linear to near-circularly polarized light, while the laser intensity for each case only differs by $6 \%$ percent. This indicates that the change in the velocity and thus the change in the dipole force are due to field-induced alignment. We also measured the velocity shifts for each polarization using a molecular beam seeded with 450 mbar of $\mathrm{CS}_{2}$ where rotational cooling is not effective. The rotational temperature is sufficiently high so that the measured velocity shifts show no polarization dependence, both with a peak velocity shift of approximately $7.3 \mathrm{~ms}^{-1}$. These measurements are shown in Fig. 3(c), and show that the change in velocity in Fig. 3(b) is due to laser-induced alignment. These data also allowed us to reduce the uncertainty in the peak intensity of the IR beam by fitting the intensity using the average polarizability, and we obtain an intensity of $5.7 \pm 0.3 \times 10^{11} \mathrm{~W} \mathrm{~cm}^{-2}$ for the linearly polarized case and $6 \%$ less for the circularly polarized case, both within the uncertainty of our initial measurement of peak intensity. The velocity at a specific temperature and intensity is determined by numerically solving the classical equation of motion of a molecule in the optical field from $F=m \frac{d^{2} r}{d t^{2}}=-\nabla V(r, t)$. By relating the amplitude of the electric field $E(r, t)$ to the laser intensity $I(r, t)$, the optical potential is $V(r, t)=-\frac{1}{2} \frac{\overline{\alpha_{\text {eff }}(r, t)}}{\epsilon_{0} c} I(r, t)$ and the force is dependent on both the spatial intensity gradient and the effective polarizability. For a Gaussian intensity profile $I(x, t)=g(t) I_{0} e^{-\left(2 x^{2} / \omega^{2}\right)}$, the force along the molecular beam axis can be expressed as $F(x, t)=$ $-\frac{2 x g(t) I_{0}}{c \epsilon_{0} \omega^{2}} e^{-\left(2 x^{2} / \omega^{2}\right)}\left[\overline{\alpha_{\text {eff }}(I)}+\frac{d \overline{\alpha_{\text {eff }}(I)}}{d I} e^{-\left(2 x^{2} / \omega^{2}\right)}\right]$ where $\omega$ is the $e^{-2}$ Gaussian spot size of the beam and $g(t)$ is the temporal profile of the laser pulse. In the absence of alignment $\frac{d \overline{\mathrm{efff}_{\mathrm{ef}}(I)}}{d I}=0$, and the force, $F(x, t)$, reduces to the more familiar expression $[1,19]$. Based on simulations of the induced velocity over a range of rotational temperatures a best fit to our data is obtained at $35 \pm 10 \mathrm{~K}$. The average effective polarizability at $I_{0}=5.7 \times 10^{11} \mathrm{~W} \mathrm{~cm}^{-2}$ and $T=35 \mathrm{~K} \quad$ is $\quad \bar{\alpha}_{\text {eff }}=11.4 \times 10^{-40} \mathrm{C} \mathrm{m}^{2} \mathrm{~V}^{-1} \quad$ and $\overline{\left\langle\cos ^{2} \theta_{\text {lin }}\right\rangle}=0.49$ for linearly polarized light and $\bar{\alpha}_{\text {eff }}=$ $10.1 \times 10^{-40} \mathrm{C} \mathrm{m}^{2} \mathrm{~V}^{-1}$ with $\overline{\left\langle\cos ^{2} \theta_{\text {circ }}\right\rangle}=0.27$ for circularly polarized light. The average polarizabilty of the molecule not aligned by the field is $9.7 \times$ $10^{-40} \mathrm{C} \mathrm{m}^{2} \mathrm{~V}^{-1}$ with $\left\langle\cos ^{2} \theta\right\rangle=\frac{1}{3}$. The optical well depths 


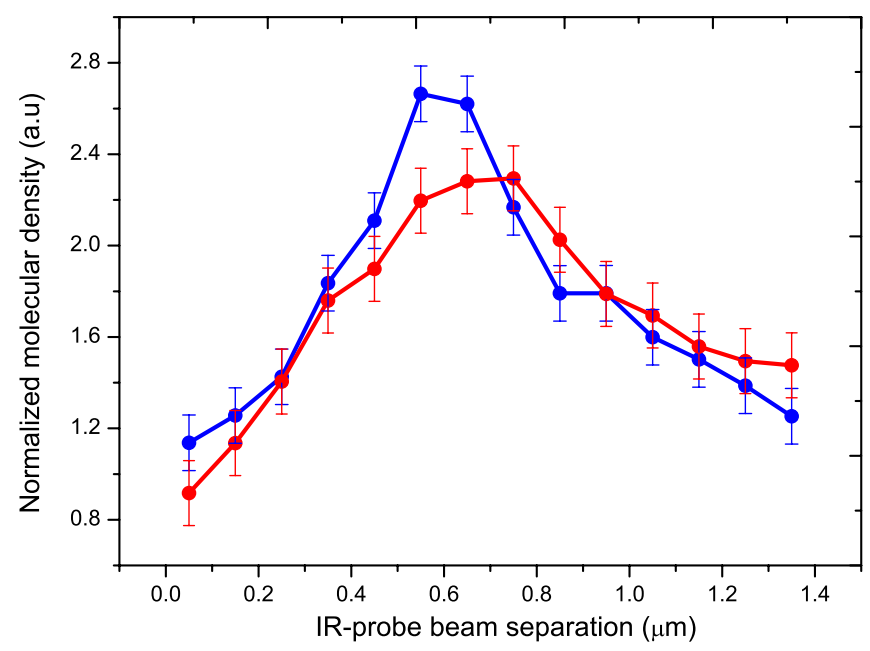

FIG. 4 (color online). Molecular density in the focal region of the molecular-optical lens along the $x$ axis. The linearly polarized light [dark gray (blue) circles] produces a focal length of $600 \mu \mathrm{m}$, and the circularly polarized light [light gray (red) circles] produces a focal length of $700 \mu \mathrm{m}$.

for linear and near-circular polarized fields are $89 \mathrm{~K}$ and $74 \mathrm{~K}$, respectively, producing a $25 \%$ change in the dipole force. When corrected for the lower intensity of the circularly polarized beam (red dashed line on Fig. 3(b)], a 20\% change in dipole force is produced between the laser polarizations at $I_{0}=5.7 \times 10^{11} \mathrm{~W} \mathrm{~cm}^{-2}$.

The optical force in the $y$ direction acts as a cylindrical lens for the molecules [17]. This lens is present for $\sim 15 \mathrm{ns,}$ and only molecules in the field during this time are focused. Molecules focus closer to the IR beam for linear polarization when compared to circularly polarized light. This is shown in Fig. 4 as a plot of density (ionization signal) distance between the center of IR focus and the probe beam. A shorter $600 \mu \mathrm{m}$ focal length lens is produced for the linearly polarized field when compared to $700 \mu \mathrm{m}$ for the circularly polarized light. The numerical aperture of a conventional optical lens is $\approx D / 2 f$, where $D$ is the lens diameter and $f$ is the focal length. A larger numerical aperture produces a smaller focused spot and a higher on-axis intensity. Our lens has a higher numerical aperture for linearly polarized light than for circularly polarized light, and we expect a smaller molecular focus and a higher density for linearly polarized light. This is seen in Fig. 4 where the peak density, normalized to the background gas density, is $21 \%$ larger for the linearly polarized case than for the near-circularly polarized beam. This indicates that the spot size is smaller by approximately the same amount.

We have measured the effect of laser-induced molecular alignment on the dipole force for the linear molecule $\mathrm{CS}_{2}$. We have measured a $25 \%$ change in the peak velocity imparted to molecules via the dipole force using a light field of two different polarizations corresponding to a $20 \%$ in the dipole force when corrected for the $6 \%$ change in laser intensity between the two polarizations. Our measurements demonstrate that laser-induced alignment can be used to modify the dipole force. This new means of tailoring the dipole force has applications in the focusing of molecules onto surfaces and for molecular separation schemes based on polarizability-to-mass ratio. As alignment, and therefore the force, is dependent on the rotational state, the variation of dipole force with rotational state could be used for rotational state selection of nonpolar molecules. Such a scheme could be applied to molecules that are not paramagnetic or do not have permanent electric dipole moment. This differential force will be particularly beneficial to the optical Stark deceleration method where separation of rotationally cold molecules will be required to achieve ultralow temperatures through evaporative cooling or sympathetic cooling with cold atoms [29].

We acknowledge useful discussions with J. Underwood.

[1] H. Stapelfeldt et al., Phys. Rev. Lett. 79, 2787 (1997).

[2] J. E. Bjorkholm et al., Phys. Rev. Lett. 41, 1361 (1978).

[3] T. Weber et al., Science 299, 232 (2003).

[4] A. Ashkin, Proc. Natl. Acad. Sci. U.S.A. 94, 4853 (1997).

[5] M. D. Wang et al., Science 282, 902 (1998).

[6] F. Buajarern et al., J. Chem. Phys. 125, 114506 (2006).

[7] H. Sakai et al., J. Chem. Phys. 110, 10235 (1999).

[8] J. J. Larson et al., J. Chem. Phys. 111, 7774 (1999).

[9] J. G. Underwood, B. J. Sussman, and A. Stolow, Phys. Rev. Lett. 94, 143002 (2005).

[10] J. J. Larsen et al., Phys. Rev. Lett. 85, 2470 (2000).

[11] Z. Vager, R. Naaman, and E. Kanter, Science 244, 426 (1989).

[12] J. Itatani et al., Nature (London) 432, 867 (2004).

[13] C. Z. Bisgaard et al., Science 323, 1464 (2009).

[14] J.C.H. Spence and R.B. Doak, Phys. Rev. Lett. 92, 198102 (2004).

[15] E. R. Peterson et al., Appl. Phys. Lett. 92, 094106 (2008).

[16] R. Fulton, A. I. Bishop, and P. F. Barker, Phys. Rev. Lett. 93, 243004 (2004).

[17] B. S. Zhao et al., J. Chem. Phys. 119, 8905 (2003).

[18] H. Sakai et al., Phys. Rev. A 57, 2794 (1998).

[19] H. S. Chung et al., J. Chem. Phys. 114, 8293 (2001).

[20] B. S. Zhao et al., Phys. Rev. Lett. 85, 2705 (2000).

[21] H. T. Bookey, M. N. Shneider, and P. F. Barker, Phys. Rev. Lett. 99, 133001 (2007).

[22] J. Ortigoso et al., J. Chem. Phys. 110, 3870 (1999).

[23] E. Hamilton et al., Phys. Rev. A 72, 043402 (2005).

[24] T. Seideman and E. Hamilton, Adv. At. Mol. Opt. Phys. 52, 289 (2006).

[25] T. Seideman, J. Chem. Phys. 107, 10420 (1997).

[26] C. Cossart-Magos et al., J. Chem. Phys. 104, 7412 (1996).

[27] J. Baker, M. Konstantaki, and S. Couris, J. Chem. Phys. 103, 2436 (1995).

[28] R. A. Morgan et al., J. Chem. Phys. 104, 6117 (1996).

[29] P. Barletta, J. Tennyson, and P. F. Barker, Phys. Rev. A 78, 052707 (2008). 